\title{
SUBLINEAR UPPER BOUNDS FOR STOCHASTIC PROGRAMS WITH RECOURSE
}

\author{
John R. BIRGE \\ Department of Industrial and Operations Engineering, University of Michigan, Ann Arbor, MI 48109, \\ USA
}

\author{
Roger J.-B. WETS \\ Department of Mathematics, University of California, Davis, CA 95616, USA
}

Received 31 October 1986

Revised manuscript received 11 January 1988

\begin{abstract}
Separable sublinear functions are used to provide upper bounds on the recourse function of a stochastic program. The resulting problem's objective involves the inf-convolution of convex functions. A dual of this problem is formulated to obtain an implementable procedure to calculate the bound. Function evaluations for the resulting convex program only require a small number of single integrations in contrast with previous upper bounds that require a number of function evaluations that grows exponentially in the number of random variables. The sublinear bound can often be used when other suggested upper bounds are intractable. Computational results indicate that the sublinear approximation provides good, efficient bounds on the stochastic program objective value.
\end{abstract}

Key words: Stochastic programming, sublinear function, simple recourse problem, recourse model, duality, approximation.

\section{Introduction}

We consider the following stochastic program with recourse:

$$
\begin{aligned}
& \text { find } x \in \mathbb{R}^{n_{1}}, \chi \in \mathbb{R}^{m_{2}} \text { such that } A x=b, T x-\chi=0, x \geqslant 0 \text {, } \\
& \text { and } z=c x+\Psi(\chi) \text { is minimized, }
\end{aligned}
$$

where $\Psi(\chi)=E\{\psi(\chi, \xi)\}$ and $\psi(\chi, \xi)$ is the recourse function defined by

$$
\psi(\chi, \xi)=\inf _{y \in \mathbb{R}_{+}^{n}}\{q y \mid W y=\xi-\chi\} .
$$

This research has been partially supported by the National Science Foundation. The first author's work was also supported in part by Office of Naval Research Grant N00014-86-K-0628 and by the National Research Council under a Research Associateship at the Naval Postgraduate School, Monterey, California. 
The random $m_{2}$-vector $\xi$ is defined on a probability space $(\Xi, \mathscr{B}, \mathscr{P})$. The vectors $b \in \mathbb{R}^{m_{1}}, c \in \mathbb{R}^{n_{1}}, q \in \mathbb{R}^{n_{2}}$, and matrices, $A \in \mathbb{R}^{m_{1} \times n_{1}}, T \in \mathbb{R}^{m_{2} \times n_{1}}, W \in \mathbb{R}^{m_{2} \times n_{2}}$, are deterministic. In more general models, $q$ and $T$ may be stochastic, but we confine our attention to the case of random right-hand sides in (1.2).

Difficulties in performing the multiple integration to evaluate $\Psi(\chi)$ make the solution of (1.1) especially complex. Solution procedures that do not assume special structure, therefore, involve some approximation to $\Psi(\chi)$ and its derivatives. One type of procedure is to sample from $\xi$ randomly and to use sample information to guide an optimization algorithm. These stochastic quasi-gradient methods (see Ermoliev, 1983; and Ermoliev and Gaivoronski, 1987) have asymptotic convergence properties, but they are limited by a lack of computable bounds.

Other approximation procedures for (1.1) provide bounds by discretizing $\Xi$ (Huang, Ziemba and Ben-Tal, 1977; Kall and Stoyan, 1982; Birge and Wets, 1986a). Lower and upper bounds are used to provide termination criteria for solution algorithms (Birge, 1983). The lower bounds are based on Jensen's inequality and, in general, require a small number of function evaluations. The upper bounds are, however, obtained by extremal measures that require evaluating $\psi(\chi, \cdot)$ at all extreme points of $\Xi$ (or some region containing $\Xi$ ), i.e., at least $2^{m_{2}}$ solutions of (1.2) with varying $\xi$. These approaches are then limited to problems with small $m_{2}$. The sublinear upper bound we propose requires only solving $O\left(m_{2}\right)$ linear programs (1.2), so that a much broader class of problems can be considered.

Various methods have been suggested for solving the large-scale linear program associated with a discretization of $\Xi$ (Wets, 1987). These methods include basis factorization (Strazicky, 1980; Kall, 1979), inner linearization (Dantzig and Madansky, 1961), and outer linearization (Van Slyke and Wets, 1969). Implementations are described in Kall and Keller (1983) and Birge (1985, 1987). In general, the number of realizations in the discretization is limited to numbers in the hundreds (i.e., $m_{2}<10$ ). The discretization level is not always enough to provide close bounds on the objective value. Solution times can also become extremely long. The situation is better, however, if (1.1) has special structure.

The stochastic program with simple recourse is a special instance of (1.1) in which $W=(I,-I)$, where $I$ is an $m_{2} \times m_{2}$ identity matrix. This allows for the use of general nonlinear optimization procedures (Nazareth and Wets, 1986) and combinations of linear and nonlinear procedures $(\mathrm{Qi}, 1986)$. Separability reduces the $\boldsymbol{m}_{2}$-dimensional integration to $m_{2}$ independent one-dimensional integrals; it renders possible the direct computation of the values and the derivatives of $\Psi$ for use in optimization.

We obtain efficient upper bounds by replacing the recourse function with functions similar to simple recourse functions. We generalize and extend the ray function approximation in Birge and Wets (1986a) and the efficient network implementation in Wallace (1987). We show that linear transformations of the random vectors can be used to obtain a variety of separable, sublinear bounding functions. We also give a dual-based solution procedure for combining these bounding functions. The separable sublinear functions allow for monotropic optimization (Rockafellar, 1984). 
The convex hull of several of these functions is used in the calculation of the upper bound. In our experience, the solution of this upper bounding problem provides quick solutions that are close in value to the optimum. It may be especially valuable in providing initial solution values (as in Birge and Wets, 1984) for further optimization procedures (Nazareth and Wets, 1986).

The simple recourse problem and its properties are described in Section 2. Section 3 describes the sublinear approximation procedure and its relation to the simple recourse problem. Section 4 explains how the sublinear approximation is solved using its dual, and Section 5 discusses our implementation. Section 6 presents computational results. Section 7 discusses possible extensions.

\section{The simple recourse problem}

The simple recourse problem has a separable recourse function that is written as $\Psi(\chi)=\sum \Psi_{i}\left(\chi_{i}\right)$ where $\Psi_{i}\left(\chi_{i}\right)=\int \psi_{i}\left(\chi_{i}, \xi_{i}\right) \mathrm{d} P_{i}\left(\xi_{i}\right), P_{i}$ is the marginal distribution function of $\xi_{i}$, which we assume has bounded first and second moments, and

$$
\begin{aligned}
\psi_{i}\left(\chi_{i}, \xi_{i}\right) & =\inf \left\{q_{i}^{+} y_{i}^{+}+q_{i}^{-} y_{i}^{-} \mid y_{i}^{+}-y_{i}^{-}=\xi_{i}-\chi_{i}, y_{i}^{+} \geqslant 0, y_{i}^{-} \geqslant 0\right\} \\
& = \begin{cases}q_{i}^{+}\left(\xi_{i}-\chi_{i}\right) & \text { if } \chi_{i} \leqslant \xi_{i}, \\
q_{i}^{-}\left(\chi_{i}-\xi_{i}\right) & \text { if } \chi_{i}>\xi_{i} .\end{cases}
\end{aligned}
$$

Detailed properties of the simple recourse problem are given in Wets $(1966,1974 \mathrm{a})$ and Parikh (1968).

The simple recourse function components $\Psi_{i}\left(\chi_{i}\right)$ can then be written as

$$
\Psi_{i}\left(\chi_{i}\right)=q_{i}^{+} \bar{\xi}_{i}-q_{i}^{+} \chi_{i}+q_{i} \chi_{i} P_{i}\left(\chi_{i}\right)-q_{i} \int_{-\infty}^{\chi_{i}} \xi_{i} \mathrm{~d} P_{i}\left(\xi_{i}\right),
$$

where $q_{i}=q_{i}^{+}+q_{i}^{-}>0$ and $\bar{\xi}_{i}=\int \xi_{i} \mathrm{~d} P_{i}\left(\xi_{i}\right)$. The functions $\chi \mapsto \Psi(\chi)$ and $\chi_{i} \mapsto \Psi_{i}\left(\chi_{i}\right)$ are continuous convex functions on $\mathbb{R}^{m_{2}}$ and $\mathbb{R}$, respectively (Wets, 1974a). The subdifferential at $\chi_{i}$ is given by

$$
\partial \Psi_{i}\left(\chi_{i}\right)=\left\{\pi \mid-q_{i}^{+}+q_{i} P_{i}^{-}\left(\chi_{i}\right) \leqslant \pi \leqslant-q_{i}^{+}+q_{i} P_{i}\left(\chi_{i}\right)\right\},
$$

where $P_{i}^{-}\left(\chi_{i}\right)=\lim _{y \uparrow \chi_{i}} P_{i}(y)$ (Wets, 1974a). From (2.3), if $P_{i}$ is continuous, then $\Psi_{i}$ is differentiable.

The development of our sublinear approximation relies upon the use of conjugate functions. To find the conjugate of $\Psi_{i}$, first define

$$
G_{i}(p)=\left\{y \mid P_{i}^{-}(y) \leqslant p \leqslant P_{i}(y)\right\}
$$

The conjugate of $\Psi_{i}$ is written

$$
\Psi_{i}^{*}\left(v_{i}\right)=\sup _{\chi_{i}}\left\{v_{i} \chi_{i}-\Psi_{i}\left(\chi_{i}\right)\right\}
$$


Proposition 2.1. The conjugate function of $\Psi_{i}$ defined in (2.2) is given by

$$
\Psi_{i}^{*}\left(v_{i}\right)= \begin{cases}-q_{i}^{+} \bar{\xi}_{i}+\left(v_{i}+q_{i}^{+}\right) y-q_{i} y P_{i}(y)+q_{i} \int_{-\infty}^{y} \xi_{i} \mathrm{~d} P_{i}\left(\xi_{i}\right), & \text { if }-q_{i}^{+}<v_{i}<q_{i}^{-}, \\ -q_{i}^{+} \bar{\xi}_{i} & \text { if } v_{i}=-q_{i}^{+}, \\ q_{i}^{-} \bar{\xi}_{i} & \text { if } v_{i}=q_{i}^{-}, \\ +\infty, & \text { otherwise, }\end{cases}
$$

where $y \in G_{i}\left(\left(v_{i}+q_{i}^{+}\right) / q_{i}\right)$.

Proof. From (2.5) and (2.2), we have

$$
\begin{aligned}
\Psi_{i}^{*}\left(v_{i}\right) & =\sup _{\chi_{i}}\left\{v_{i} \chi_{i}-q_{i}^{+} \bar{\xi}_{i}+q_{i}^{+} \chi_{i}-q_{i} \chi_{i} P_{i}\left(\chi_{i}\right)+q_{i} \int_{-\infty}^{x_{i}} \xi_{i} \mathrm{~d} P_{i}\left(\xi_{i}\right)\right\} \\
& =-q_{i}^{+} \bar{\xi}_{i}+\sup \left\{\left(v_{i}+q_{i}^{+}\right) \chi_{i}-q_{i} \chi_{i} P_{i}\left(\chi_{i}\right)+q_{i} \int_{-\infty}^{\chi_{i}} \xi_{i} \mathrm{~d} P_{i}\left(\xi_{i}\right)\right\} .
\end{aligned}
$$

From $(2.7)$, it is clear that $\Psi_{i}^{*}\left(v_{i}\right)=+\infty$ if $v_{i}<-q_{i}^{+}$or $v_{i}>q_{i}^{-}$. We, therefore, assume that $-q_{i}^{+} \leqslant v_{i} \leqslant q_{i}^{-}$. For $v_{i} \in\left(-q_{i}^{+}, q_{i}^{-}\right)$, the set of $\chi_{i}$ that attains the supremum in (2.7) is $\left\{\chi_{i} \mid P_{i}^{-}\left(\chi_{i}\right) \leqslant\left(v_{i}+q_{i}^{+}\right) / q_{i} \leqslant P_{i}\left(\chi_{i}\right)\right\}=G_{i}\left(\left(v_{i}+q_{i}^{+}\right) / q_{i}\right)$. Substitution of $y \in$ $G_{i}\left(\left(v_{i}+q_{i}^{+}\right) / q_{i}\right)$ yields the first formula in (2.6). For $v_{i}$ on the boundary of $\left[-q_{i}^{+}\right.$, $\left.q_{i}^{-}\right]$and bounded distributions, the same argument applies. The formulas for unbounded distributions follow directly given our assumption of bounded first and second moments.

The following corollaries follow immediately from Proposition 2.1.

Corollary 2.2. If $P_{i}$ is continuous, then

$$
\Psi_{i}^{*}\left(v_{i}\right)= \begin{cases}-q_{i}^{+} \bar{\xi}_{i}+q_{i} \int_{-\infty}^{y} \xi_{i} \mathrm{~d} P_{i}\left(\xi_{i}\right) & \text { if }-q_{i}^{+}<v_{i}<q_{i}^{-}, \\ -q_{i}^{+} \bar{\xi}_{i} & \text { if } v_{i}=-q_{i}^{+}, \\ q_{i}^{-} \bar{\xi}_{i} & \text { if } v_{i}=q_{i}^{-} \\ +\infty, & \text { otherwise, }\end{cases}
$$

where $y \in G_{i}\left(\left(v_{i}+q_{i}^{+}\right) / q_{i}\right)$.

Corollary 2.3. If $\xi_{i}$ has the degenerate distribution

$$
P_{i}\left(\xi_{i}\right)= \begin{cases}1 & \text { if } \xi_{i} \geqslant \bar{\xi}_{i}, \\ 0 & \text { otherwise, }\end{cases}
$$

then

$$
\Psi_{i}^{*}\left(v_{i}\right)=\left\{\begin{aligned}
v_{i} \bar{\xi}_{i} & \text { if }-q_{i}^{+} \leqslant v_{i} \leqslant q_{i}^{-}, \\
+\infty & \text { otherwise. }
\end{aligned}\right.
$$


In addition to $\Psi_{i}^{*}$, we are also interested in its subgradients.

Proposition 2.4. The subdifferential of $\Psi_{i}^{*}$ at $v_{i} \in \operatorname{ri}\left(\operatorname{dom} \Psi_{i}^{*}\right)$ (the relative interior of the effective domain of $\Psi_{i}^{*}$ ) is given by

$$
\partial \Psi_{i}^{*}\left(v_{i}\right)=G_{i}\left(\frac{v_{i}+q_{i}^{+}}{q_{i}}\right) .
$$

Proof. The subgradients are found directly using (2.6).

\section{The sublinear upper bound}

Simple recourse functions are used here to approximate the general recourse function $\psi$ as defined in (1.2). We assume that $\psi(\chi, \xi)$ is finite for all values of $\chi$ and $\xi$, i.e., pos $W=\mathbb{R}^{m_{2}}$. This assumption corresponds to complete recourse in stochastic programming. In practice, it can be achieved by introducing appropriate penalties in the recourse problem. In this case, the function $\phi$ defined by

$$
\phi(\xi-\chi)=\psi(\chi, \xi)
$$

is sublinear (positively homogeneous and convex). This property allows the simple recourse function approximation. The function $\phi$ is also polyhedral (i.e., its epigraph is a polyhedral cone).

Birge and Wets (1986a) introduced a method for approximating $\psi$ by simple recourse functions. This method was based on solving the linear program:

find $y \in \mathbb{R}^{n_{2}}$ such that $W y=e_{i}, y \geqslant 0$, and $q y$ is minimized,

where $e_{i}$ is the $i$ th unit $m_{2}$-vector. The optimal solution value of (3.2) is $q_{I(i)}^{+}$. If we substitute $-e_{i}$ for $e_{i}$, the optimal solution value is $-q_{I(i)}^{-}$. By sublinearity,

$$
\psi(\chi, \xi) \leqslant \psi_{I}(\chi, \xi)=\sum_{i=1}^{m_{2}} \psi_{I(i)}\left(\chi_{i}, \xi_{i}\right)
$$

where

$$
\psi_{I(i)}\left(\chi_{i}, \xi_{i}\right)= \begin{cases}q_{I(i)}^{+}\left(\xi_{i}-\chi_{i}\right), & \xi_{i} \geqslant \chi_{i}, \\ q_{I(i)}^{-}\left(\chi_{i}-\xi_{i}\right), & \xi_{i}<\chi_{i} .\end{cases}
$$

The function $\psi_{I}$ is a simple recourse function.

By integration in (3.3), we have

$$
\Psi(\chi) \leqslant \Psi_{I}(\chi)
$$

where

$$
\begin{aligned}
\Psi_{I}(\chi) & =\int_{\Xi} \psi_{I}(\chi, \xi) \mathrm{d} P(\xi) \\
& =\sum_{i=1}^{m_{2}}\left\{\int_{\xi_{i} \geqslant \chi_{i}} q_{I(i)}^{+}\left(\xi_{i}-\chi_{i}\right) \mathrm{d} P\left(\xi_{i}\right)+\int_{\xi_{i}<x_{i}} q_{I(i)}^{-}\left(\chi_{i}-\xi_{i}\right) \mathrm{d} P\left(\xi_{i}\right)\right\} .
\end{aligned}
$$


Note that $\Psi_{I}$ is separable in the components of $\chi$ and only line integration is required in its computation.

Other approximations are obtained by considering directions other than $\pm e_{i}$ in (3.2). Let $h_{1}, \ldots, h_{K} \in \mathbb{R}^{m_{2}}$ positively $\operatorname{span} \mathbb{R}^{m_{2}}$ (i.e., $\mathbb{R}^{m_{2}}=\operatorname{pos}\left[h_{1}, \ldots, h_{K}\right]$ ). Substitute $h_{j}, j=1, \ldots, K$, for $e_{i}$ in (3.2) and let the optimal solution values be $q_{H(j)}, j=$ $1, \ldots, K$. We then have

$$
\psi(\chi, \xi) \leqslant \inf \left\{\sum_{j=1}^{K} \lambda_{j} q_{H(j)} \mid \sum_{j=1}^{K} \lambda_{j} h_{j}=\xi-\chi, \lambda_{j} \geqslant 0, j=1, \ldots, K\right\} .
$$

If $H=\left[h_{1}, \ldots, h_{K}\right]$ includes all columns of $W$, then (3.5) becomes an equality (see Birge and Wets, 1986b).

A difficulty in using (3.5) for approximating $\Psi$ is that in general an optimization must still be performed inside the integral. This solution is immediate, however, if $H$ is a positive linear basis for $\mathbb{R}^{m_{2}}$, i.e., every point in $\mathbb{R}^{m_{2}}$ corresponds to a unique positive combination of the $h_{i}$. A convenient choice for such a set is to use a linear basis $D=\left[d_{1}, \ldots, d_{m_{2}}\right]$ for $\mathbb{R}^{m_{2}}$ and $-D=\left[-d_{1}, \ldots,-d_{m_{2}}\right]$, so that $\operatorname{pos}[D,-D]=$ $\mathbb{R}^{m_{2}}$. In this case, $\xi-\chi=\sum_{i=1}^{m_{2}}\left(D^{-1}\right)_{i} \cdot(\xi-\chi) d_{i}$, where $\left(D^{-1}\right)_{i}$. indicates the $i$ th row of $D^{-1}$. The approximation is

$$
\psi(\chi, \xi) \leqslant \psi_{D}(\chi, \xi)=\sum_{i=1}^{m_{2}} \psi_{D(i)}(\chi, \xi)
$$

where

$$
\psi_{D(i)}(\chi, \xi)= \begin{cases}q_{D(i)}^{+}\left(D^{-1}\right)_{i} \cdot(\xi-\chi) & \text { if }\left(D^{-1}\right)_{i \cdot}(\xi-\chi) \geqslant 0 \\ q_{D(i)}^{-}\left(D^{-1}\right)_{i} \cdot(\chi-\xi) & \text { if }\left(D^{-1}\right)_{i \cdot} \cdot(\chi-\xi)>0\end{cases}
$$

and $q_{D(i)}^{+}$and $q_{D(i)}^{-}$are the optimal solution values of (3.2) with $d_{i}$ and $-d_{i}$, respectively, substituted for $e_{i}$ and $-e_{i}$.

Several different bases are used in the sublinear approximation of $\psi$. For $\mathscr{D}=$ $\left\{D^{1}, \ldots, D^{L}\right\}$, a set of linear bases for $\mathbb{R}^{m_{2}}$,

$$
\begin{aligned}
\psi(\chi, \xi) & \leqslant \inf \left\{\sum_{j=1}^{L} \lambda^{j} \psi_{D^{j}}\left(\chi, \xi^{j}\right) \mid \sum_{j=1}^{L} \lambda^{j} \xi^{j}=\xi, \sum_{j=1}^{L} \lambda^{j}=1, \lambda^{j} \geqslant 0, \text { for all } j\right\}, \\
& =\operatorname{co}\left(\psi_{D^{j}}(\chi, \cdot), j=1, \ldots, L\right)(\xi)
\end{aligned}
$$

the function obtained by taking the convex hull of the epigraphs of $\psi_{D}, j=1, \ldots, L$. Of course, if $\mathscr{D}$ includes all linear bases in $W$, then (3.8) is satisfied as an equality. Although it might be simpler than (3.5), inequality (3.8) is still difficult to use computationally, again because of the minimization required inside the integral. A weaker, but usable inequality is obtained by reversing the infimum and integration. For this, we define

$$
\Psi_{D}(\chi)=\sum_{i=1}^{m_{2}} \Psi_{D(i)}(\chi)
$$


where

$$
\begin{aligned}
\Psi_{D(i)}(\chi)= & \int_{\left(D^{-1}\right)_{i} \cdot(\xi-\chi) \geq 0} q_{D(i)}^{+}\left(D^{-1}\right)_{i \cdot}(\xi-\chi) \mathrm{d} P(\xi) \\
& +\int_{\left(D^{-1}\right)_{i} \cdot(\xi-\chi)<0} q_{D(i)}^{-}\left(D^{-1}\right)_{i \cdot}(\chi-\xi) \mathrm{d} P(\xi) .
\end{aligned}
$$

Proposition 3.1. Let $\mathscr{D}$ be a set of linear bases of $\mathbb{R}^{m_{2}}$, then

$$
\Psi(\chi) \leqslant \operatorname{co}\left\{\Psi_{D}, D \in \mathscr{D}\right\}(\chi)
$$

Proof. Integration of (3.6) yields

$$
\Psi(\chi) \leqslant \Psi_{D}(\chi)
$$

for any $D \in \mathscr{D}$. So, $\sum_{i=1}^{m_{2}} \lambda^{i} \Psi\left(\chi^{i}\right) \leqslant \sum_{i=1}^{m_{2}} \lambda^{i} \Psi_{D}\left(\chi^{i}\right)$, for any $\lambda^{i} \geqslant 0$. Letting $\sum_{i=1}^{m_{2}} \lambda^{i}=1$ and $\chi=\sum_{i=1}^{m_{2}} \lambda^{i} \chi^{i}$ yields $(3.10)$ by the convexity of $\Psi$.

Equality in (3.10) can only be guaranteed in very special cases, even if $\mathscr{D}$ includes all linear bases from $W$. To see this simply observe that if $\mathscr{D}$ is rich enough, then

$$
\int \operatorname{co} \Psi_{D}=\int \inf _{D} \Psi_{D} \geqslant \inf _{D} \int \Psi_{D}
$$

with strict inequality except in degenerate cases such as: $\mathscr{D}$ is a singleton, the $\Psi_{D}$ 's are linear, the probability measure is degenerate, etc. But as suggested by Proposition $3.1, \operatorname{co}\left\{\Psi_{D}, D \in \mathscr{D}\right\}(\chi)$ always provides us with an upper bound, which we call the sublinear upper bound. This bound is relatively easy to compute, even when extremal measure procedures require more computations than can be implemented in realistic times. For example, for ten random variables, the basic sublinear upper bound requires the solutions of twenty linear programs. Extremal measure methods require the solutions of more than one thousand linear programs for a single bound in this case.

We have also observed that the solution obtained from solving the "stochastic" program

$$
\begin{aligned}
& \text { find } x \in \mathbb{R}^{n_{1}}, \chi \in \mathbb{R}^{m_{2}} \text { such that } A x=b, T x=\chi, x \geqslant 0 \text {, } \\
& \text { and } z=c x+\operatorname{co}\left\{\Psi_{D}, D \in \mathscr{D}\right\}(\chi) \text { is minimized, }
\end{aligned}
$$

instead of (1.1), is usually a very good approximation of the optimal solution, much better than may be expected from the relatively lax inequality (3.10). Our experience shows that the function $\operatorname{co}\left\{\Psi_{D}, D \in \mathscr{D}\right\}$ is "parallel" to $\Psi$, i.e.,

$$
\partial \Psi(\chi) \approx \partial \operatorname{co}\left\{\Psi_{D}, D \in \mathscr{D}\right\}(\chi)
$$

In the Appendix, we provide a heuristic argument and small example. 


\section{Dualization and solution procedures}

Several questions must be answered in order to solve (1.1) with $\operatorname{co}\left\{\Psi_{D}, D \in \mathscr{D}\right\}$ substituted for $\Psi$. The first concern is that finding the convex hull of a set of functions is itself a difficult task. The second problem is to evaluate $\Psi_{D}$ efficiently. A third area involves the choice of $\mathscr{D}$. We address each of these problems in this section. After substituting for $\Psi,(1.1)$ becomes:

$$
\begin{aligned}
\text { find } x \in \mathbb{R}^{n_{1}}, \chi \in \mathbb{R}^{m_{2}} \text { such that } A x=b, T x-\chi=0, x \geqslant 0, \\
\text { and } z=c x+\operatorname{co}\left\{\Psi_{D}, D \in \mathscr{D}\right\}(\chi) \text { is minimized. }
\end{aligned}
$$

Instead of solving (4.1), we consider a dual program to (4.1). The dual program has a computational advantage because the convex hull operation is replaced by a supremum.

Proposition 4.1. A dual program to (4.1) is given by

$$
\text { find } \sigma \in \mathbb{R}^{m_{1}}, \pi \in \mathbb{R}^{m_{2}} \text { such that } \sigma A+\pi T \leqslant c
$$

$$
\text { and } w=\sigma b-\left(\sup _{D \in \mathscr{D}} \Psi_{D}^{*}\right)(-\pi) \text { is maximized, }
$$

where $\Psi_{D}^{*}$ is the conjugate function of $\Psi_{D}$ and where the optimal value of (4.2), $w^{*}=z^{*}$, the optimal value of (4.1).

Proof. A general dual of (4.1) (see, e.g., Rockafellar, 1974; Geoffrion, 1971) can be written as

$$
\max _{\pi, \sigma}\left\{\inf _{x \geqslant 0, \chi} c x+g(\chi)+\sigma(b-A x)+\pi(\chi-T x)\right\}
$$

where $g(\chi)=\operatorname{co}\left\{\Psi_{D} \mid D \in \mathscr{D}\right\}(\chi)$. Since (4.1) involves linear constraints, the optimal values of (4.3) and (4.1) are the same. We can rewrite (4.3) as

$$
\max _{\pi, \sigma}\left\{\inf _{x \geqslant 0, \chi}(c-\sigma A-\pi T) x-(-\pi \chi-g(\chi))+\sigma b\right\},
$$

which is equivalent to

$$
\begin{aligned}
& \text { find } \sigma \in \mathbb{R}^{m_{1}}, \pi \in \mathbb{R}^{m_{2}} \text { such that } \sigma A+\pi T \leqslant c \\
& \text { and } w=\sigma b-g^{*}(-\pi) \text { is maximized. }
\end{aligned}
$$

By Theorem 16.5 of Rockafellar (1970),

$$
\left(\operatorname{co}\left\{\Psi_{D} \mid D \in \mathscr{D}\right\}(\chi)\right)^{*}=\sup \left\{\Psi_{D}^{*} \mid D \in \mathscr{D}\right\},
$$

yielding (4.2). 
A solution of the dual program (4.2) is simpler to compute than a solution of (4.1) because $g^{*}(-\pi)$ is much easier to evaluate than $g(\chi)$. We must, however, obtain an expression for $\Psi_{D}^{*}$. First, let $P_{D(i)}$ be the distribution function of $\zeta_{i}=$ $\left(D^{-1}\right)_{i} \cdot(\xi-\chi)$ and let $\bar{\zeta}_{i}=\int \zeta_{i} \mathrm{~d} P_{D(i)}\left(\zeta_{i}\right)$. Also define

$$
G_{D(i)}(p)=\left\{y \mid P_{D(i)}^{-}(y) \leqslant p \leqslant P_{D(i)}(y)\right\} .
$$

Proposition 4.2. The conjugate function of $\Psi_{D}$ is given by

$$
\Psi_{D}^{*}(v)=\sum_{i=1}^{m_{2}} \Psi_{D(i)}^{*}(v)
$$

where

$$
\Psi_{D(i)}^{*}(v)= \begin{cases}-q_{D(i)}^{+} \bar{\zeta}_{i}+\left(v D_{\cdot i}+q_{D(i)}^{+}\right) y-q_{D(i)} y P_{D(i)}(y) \\ +q_{D(i)} \int_{-\infty}^{y} \zeta_{i} \mathrm{~d} P_{D(i)}\left(\zeta_{i}\right), & \text { if }-q_{D(i)}^{+}<v D_{\cdot i}<q_{D(i)}^{-}, \\ -q_{D(i)}^{+} \bar{\zeta}_{i} & \text { if } v D_{\cdot i}=-q_{D(i)}^{+}, \\ q_{D(i)} \bar{\zeta}_{i} & \text { if } v D \cdot i=q_{D(i)}^{-}, \\ +\infty & \text { otherwise, }\end{cases}
$$

where $q_{D(i)}=q_{D(i)}^{+}+q_{D(i)}^{-}$and $y \in G_{D(i)}\left(\left(v D_{\cdot i}+q_{D(i)}^{+}\right) / q_{D(i)}\right)$.

Proof. From (3.9), observe that

$$
\Psi_{D(i)}(\chi)=\int \psi_{i}^{s}\left(\left(D^{-1} \chi\right)_{i}, \zeta_{i}\right) \mathrm{d} P_{D(i)}\left(\zeta_{i}\right)
$$

where $\psi_{i}^{s}$ is a simple recourse function as in (2.1) with $q_{D(i)}^{+}$and $q_{D^{(i)}}^{-}$substituted for $q_{i}^{+}$and $q_{i}^{-}$respectively. From (4.9), it follows that

$$
\Psi_{D}(\chi)=\sum_{i=1}^{m_{2}} \Psi_{i}^{s}\left(\left(D^{-1} \chi\right)_{i}\right)=\Psi^{s}\left(D^{-1} \chi\right),
$$

where $\Psi_{i}^{s}$ has the form of the simple recourse function in (2.2) and $\Psi^{s}$ is the simple recourse function defined for the random variables $\zeta_{1}, \ldots, \zeta_{m_{2}}$ in place of $\xi_{1}, \ldots, \xi_{m_{2}}$.

The dual of $\Psi_{D}$ is then (see Theorem 16.3 of Rockafellar, 1970)

$$
\Psi_{D}^{*}=\left(\Psi^{s} D^{-1}\right)^{*}=D^{-1}\left(\Psi^{s}\right)^{*}
$$

where

$$
D^{-1}\left(\Psi^{s}\right)^{*}(v)=\left(\Psi^{s}\right)^{*}(v D) .
$$

Applying (4.11) and (4.12) to (2.6) yields the result.

A subgradient of $\Psi_{D}^{*}$ is used in the optimization procedure. It is also calculated from the subdifferential of $\left(\Psi^{s}\right)^{*}$. 
Proposition 4.3. The subdifferential of $\Psi_{D}^{*}$ at $v \in \operatorname{ri}\left(\operatorname{dom} \Psi_{D}^{*}\right)$ is

$$
\partial\left(\Psi_{D}^{*}\right)(v)=\left\{D y \mid y \in \mathbb{R}^{m_{2}}, y_{i} \in G_{D(i)}\left(\frac{v D_{\cdot i}+q_{D(i)}^{+}}{q_{D(i)}}\right), i=1, \ldots, m_{2}\right\} .
$$

Proof. If $w^{*}$ is a subgradient of $\left(\Psi^{s}\right)^{*}$ at $w$, then

$$
(z-w) \cdot w^{*}+\left(\Psi^{s}\right)^{*}(w) \leqslant\left(\Psi^{s}\right)^{*}(z) .
$$

From (4.11) and (4.12) if $z=u D$ and $w=v D$, (4.14) becomes

$$
(v-u) D w^{*}+\Psi_{D}^{*}(v) \leqslant \Psi_{D}^{*}(u) .
$$

From Proposition $2.4, w^{*}=\left(w_{1}^{*}, \ldots, w_{m_{2}}^{*}\right)$ where $w_{i}^{*} \in G_{D(i)}\left(\left(v D_{\cdot i}+q_{i}^{D+}\right) / q_{i}^{D}\right)$, so $D y$ in (4.13) is a subgradient of $\Psi_{D}^{*}$ at $v$. A reverse argument shows that any subgradient of $\Psi_{D}^{*}$ has the form $D y$ in (4.12), proving the result.

The expressions for $\Psi_{D}^{*}$ and $\partial \Psi_{D}^{*}$ in (4.7), (4.8) and (4.13) are used in an optimization procedure for (4.2). A difficulty is that, even when each $\Psi_{D}^{*}$ is differentiable (i.e., the distribution function of $\zeta_{i}$ is strictly increasing on its support, but not necessarily continuous), the objective function in (4.2) is not necessarily differentiable. Nondifferentiable methods (see, e.g., Lemaréchal, 1978; Lemaréchal et al., 1981; Nazareth and Wets, 1986; Polak, 1987; Wolfe, 1975) can be applied to this program. We, however, transform (4.2) into a smooth optimization problem with nonlinear constraints (as suggested, for example, in Gill, Murray and Wright, 1981). The new problem becomes

$$
\begin{aligned}
& \text { find } \sigma \in \mathbb{R}^{m_{1}}, \pi \in \mathbb{R}^{m_{2}}, \theta \in \mathbb{R} \text { such that } \\
& \qquad \begin{aligned}
\sigma A+\pi T & \leqslant c, \\
\psi_{D}^{*}(-\pi)-\theta & \leqslant 0, \text { for all } D \in \mathscr{D} \text {, and }
\end{aligned} \\
& \begin{aligned}
w=\sigma b \quad-\theta & \text { is maximized. }
\end{aligned}
\end{aligned}
$$

General methods for optimization problems with nonlinear constraints can be applied to (4.16). In our examples, we use the MINOS computer code (Murtagh and Saunders, 1980) to solve (4.16). Note that (4.16) is similar in form to a master problem in a linear outer approximation algorithm (e.g., the L-shaped method), but we solve the dual and use a nonlinear outer approximation.

\section{Implementation considerations}

In solving (4.2) or (4.16) one needs to find $P_{D(i)}$, the distribution function of $\zeta_{i}$, and $G_{D(i)}$, the inverse function. If the random variables $\xi_{j}$ are independently, normally distributed with means, $\mu_{j}$, and variances, $\sigma_{j}^{2}$, then $\zeta_{i}=\left(D^{-1}\right)_{i} \cdot(\xi-\chi)$ is 
also normally distributed with mean $\sum_{j=1}^{m_{2}}\left(D^{-1}\right)_{i j}\left(\mu_{j}-\chi_{j}\right)$ and variance $\sum_{j=1}^{m_{2}}\left(D^{-1}\right)_{i j}^{2} \sigma_{j}^{2}$. (Note that degenerate and correlated random variables can also be included among the $\xi_{i}$ with $\zeta_{i}$ remaining normally distributed.) Other distributions require special schemes in order to integrate with respect to $\zeta_{i}$. In our experiments, we used normally distributed random variables because of the ease in performing these computations. Upper bounds can be obtained for other distributions by using the approaches in Birge and Wets (1986a, 1987).

Given a problem form $((4.2)$ or $(4.16))$ and a method for finding $P_{D(i)}$, the set of bases must still be chosen. For the problem of approximating $\Psi$, it appears that the matrices to include in $\mathscr{D}$ should be chosen so that the level sets of $\psi_{D}$ cover high probability regions of the level sets of $\psi$. This coverage, however, depends on $\chi$, so the choices should be good for a range of values of $\chi$ (that would ideally include an optimal value of $\chi$ ). In our experience, the identity provided a good starting basis, especially when an optimal $\chi$ was close to $\bar{\xi}$.

We implemented several basis generation procedures that started with the identity as the first basis and then included additional bases. Generating random bases from the set of all bases proved inefficient because the corresponding functions $\Psi_{D}^{*}$ often did not improve the solution. Bases were then generated from the set of optimal bases for some $\xi$ by solving (1.2) for varying values of $\xi$ and $\chi$. A patterned choice of values for $\xi$ (using $\bar{\xi}_{i} \pm 3 \sigma_{i}$ ) proved slightly more efficient than random selection of $\xi_{i}$ and was, therefore, used in the experiments described below. Two different choices for $\chi$ were implemented. On the $k$ th solution of (4.16) with new bases added to $\mathscr{D}$, we used $\chi=\chi^{k-1}$ or $-\chi^{k-1}$ where $\chi^{k-1}$ was the optimal value of $\chi$ from the $(k-1)$ th solution of (4.16). In our experiments, $\chi=-\chi^{k-1}$ proved more effective because it included a broader class of bases by exploring different regions of $\chi$.

The sublinear upper bounding method with this implementation is given below. The basic tolerance parameters are TOLINF for infinity, TOLTERM for termination if improvements greater than TOLTERM are not found, and $L$ for the number of $\xi$ values to search over for a new basis.

\section{Sublinear Upper Bounding Method}

Step 0. Initialization. Let $D^{1}=I, K=1, \mathscr{D}=\left\{D^{1}, \ldots, D^{K}\right\}, \mathscr{I}=\emptyset, w_{\text {old }}=$ TOLINF, $l=0, \chi=0$.

Step 1. Add New Basis. Let $D^{K}=\left[D^{K}(1), \ldots, D^{K}\left(m_{2}\right)\right]$.

a. For $i=1, \ldots, m_{2}$, if $D^{K}(i) \notin \mathscr{I}$, let $\mathscr{I}=\mathscr{I} \cup D^{K}(i)$ and find $q_{D^{K}(i)}^{+}, q_{D^{K}(i)}$.

b. For $i=1, \ldots, m_{2}$, calculate parameters for $P_{D^{K}(i)}$.

If $K=1$, go to 3 .

Else, if $l=0$, choose a set of $L$ values, $\xi^{1}, \ldots, \xi^{L}$, of the random vector, $\xi$. (These may be random or according to some pattern.)

Step 2. Search for New Basis. If $l<L$, let $l=l+1$. Find $\psi\left(\chi, \xi^{l}\right)$ with an optimal basis, $D^{\prime}$.

If $D^{\prime} \notin \mathscr{D}, D^{K+1}=D^{\prime}, K=K+1$, go to 1 .

Else, go to 3 . 
Step 3. Find New Bound. Solve (4.16) to obtain $w_{\text {new }}$ and let the dual variables associated with the linear constraints be $x$. Let $\chi=T x$.

If TOLTERM $<\left(w_{\text {old }}-w_{\text {new }}\right)\left(/\left|w_{\text {new }}\right|\right.$ if $\left.w_{\text {new }} \neq 0\right)$, go to 2 .

Else, stop, $w_{\text {new }}$ is the sublinear upper bound.

Note that an upper bound is obtained at every iteration (completion of Step 3). For each iteration, the central work is in calculating $q_{D^{K}(i)}^{+}$and $q_{D^{K}(i)}^{-}$for every new basis, $D_{K}$ (assuming that $P_{D^{K}(i)}$ is easily calculated as in the normal distribution). The work of each iteration is then dominated by $\mathrm{O}\left(m_{2}\right)$ linear programs if $L$, the maximum number of trials in Step 2, is of the order $\mathrm{O}\left(m_{2}\right)$.

In our examples, we considered all $3^{m_{2}}$ combinations of $\bar{\xi}_{i}, \bar{\xi}_{i}+3 \sigma_{i}$, and $\bar{\xi}_{i}-3 \sigma_{i}$ for $i=1, \ldots, m_{2}$, because $m_{2}$ was small (at most three), and each Step 2 check was executed quickly. These additional trials would be avoided in larger problems. This option to adjust the work according to the problem does not exist in extremal measure procedures, which require function evaluations at all extreme values for a single bound. The next section presents a further comparison of the computational effort required by the sublinear and extremal measure bounds for a few examples.

\section{Numerical results}

Formulation 4.16 was used with the MINOS/AUGMENTED (MINOS Version 4.0) computer program for nonlinearly constrained problems. This implementation on The University of Michigan's Amdahl 5860 Computer found optimal solutions for (4.16) for all of the test problems tried.

The appropriate use of tolerances on constraint satisfaction was especially important in our implementation. A value of $10^{-5}$ was used for ROW TOLERANCE in the MINOS SPECS file. This allowed some flexibility in satisfying the constraints without creating large infeasibilities. To avoid infinite values of $\Psi_{D}^{*}$, the constraints

$$
-q_{D(i)}^{+} \leqslant v D_{. i} \leqslant q_{D(i)}^{-},
$$

were added to (4.16). A penalty term was included in the subgradient definition from (4.13) when (6.1) was satisfied as an equality (within the tolerance). This made the subgradient definition consistent at the boundary of the effective domain of $\Psi_{D}^{*}$.

Problem testing initially involved simple recourse problems which were solved exactly by the formulation in (4.16). After conducting this check, the method was applied to general recourse problems. The results reported here apply to the small energy decision problem in Louveaux (1987). In this problem, $m_{1}=2, n_{1}=4, m_{2}=7$, and $n_{2}=12$. Of the seven recourse problem constraints, four are balancing constraints that are fixed at zero and three are demand constraints that are stochastic. Larger problems were not considered because there was no easily available computer code that allowed for enough refinements of the bound obtained via extremal measures. 
The demands were assumed independent and normally distributed. Different problems were generated by varying the means and standard deviations of these random variables (see Table 1).

To check the accuracy of the sublinear approximation method (SUB), the test problems were also solved using the L-shaped code, NDSP (Birge, 1985), with upper bounds (EM) from the Edmundson-Madansky inequality (Madansky, 1959) and lower bounds $(\mathrm{J})$ from Jensen's inequality. Our comparison is with the EM bound because upper bounds (not just lower bounds) are needed to establish stopping conditions in an algorithm. After the $k$ th solution of the problem for EM and $\mathbf{J}$, the bounds were refined for each $\xi_{i}$ until NDSP's limit of 125 realizations of the random vector $\xi$ was reached. This occurs here when $k=4$.

The results for the EM upper bound and CPU second times for each iteration appear in Table 2. Corresponding results for SUB are also provided in Table 2. These results are intended to illustrate the behavior of the two methods on problems where both bounds can be applied. In problems with more random variables, the EM bound quickly becomes too expensive to calculate. Note, for example, that in Problem 8 after 33.80 CPU seconds, NDSP terminated because a limit of 100 L-shaped algorithm iterations (corresponding to 100 cutting planes) had been performed. Execution was not continued because of large row residuals caused by instability in the bases including these cuts (see Birge (1986) for a discussion of this phenomenon).

For the SUB results in Table 2, the algorithm is terminated when the addition of new bases does not change the solution. This is indicated by " $b$ " in Table 2 . Further improvement would be possible using groups of random variables as noted in the next section. The computation times include some iteration logging that is comparable but not identical to iteration log times included in the CPU times for EM.

A key property to note in examining Table 2 is that the sublinear method times are comparable for all problems, but the accuracy relative to the EM bounds does

Table 1

Problem parameters

\begin{tabular}{lllllll}
\hline Problem & \multicolumn{6}{c}{ Random Variables Parameters } \\
\cline { 2 - 6 } & $\mu_{1}$ & $\sigma_{1}$ & $\mu_{2}$ & $\sigma_{2}$ & $\mu_{3}$ & $\sigma_{3}$ \\
\hline 1 & 5.0 & 1.0 & 4.0 & 0.0 & 3.0 & 0.0 \\
2 & 5.0 & 0.0 & 4.0 & 1.0 & 3.0 & 0.0 \\
3 & 5.0 & 0.0 & 4.0 & 0.0 & 3.0 & 1.0 \\
4 & 5.0 & 1.0 & 4.0 & 1.0 & 3.0 & 0.0 \\
5 & 5.0 & 1.0 & 4.0 & 0.0 & 3.0 & 1.0 \\
6 & 5.0 & 0.0 & 4.0 & 1.0 & 3.0 & 1.0 \\
7 & 5.0 & 1.0 & 4.0 & 1.0 & 3.0 & 1.0 \\
8 & 2.0 & 1.0 & 4.0 & 1.0 & 3.0 & 1.5 \\
9 & 4.0 & 1.0 & 5.0 & 1.0 & 5.0 & 1.0 \\
\hline
\end{tabular}


Table 2

Results on upper bounds

\begin{tabular}{|c|c|c|c|c|c|}
\hline \multirow[t]{2}{*}{ Problem } & \multirow[t]{2}{*}{ Iteration } & \multicolumn{2}{|c|}{ EM } & \multicolumn{2}{|c|}{ SUB } \\
\hline & & Bound & CPUs $^{a}$ & Bound & CPUs \\
\hline \multirow[t]{4}{*}{1} & 1 & 395. & 0.41 & 452. & 0.82 \\
\hline & 2 & 367. & 0.75 & 385. & 1.79 \\
\hline & 3 & 366. & 1.12 & 383. & 2.38 \\
\hline & 4 & 366. & 1.27 & b & 2.75 \\
\hline \multirow[t]{4}{*}{2} & 1 & 345 . & 0.38 & 398. & 0.54 \\
\hline & 2 & 335. & 0.71 & 345. & 1.48 \\
\hline & 3 & 335. & 0.79 & $\mathrm{~b}$ & 1.71 \\
\hline & 4 & 335. & 0.88 & - & - \\
\hline \multirow[t]{4}{*}{3} & 1 & 291. & 0.42 & 304. & 1.44 \\
\hline & 2 & 289. & 0.47 & 289. & 2.03 \\
\hline & 3 & $\mathrm{~b}$ & - & $\mathrm{b}$ & 2.42 \\
\hline & 4 & - & - & - & - \\
\hline \multirow[t]{4}{*}{4} & 1 & 1180. & 0.42 & 572. & 3.37 \\
\hline & 2 & 454. & 1.01 & 440 & 5.78 \\
\hline & 3 & 425 & 1.85 & $\mathrm{~b}$ & 6.22 \\
\hline & 4 & 424. & 2.88 & - & - \\
\hline \multirow[t]{4}{*}{5} & 1 & 1140. & 0.50 & 478. & 0.63 \\
\hline & 2 & 505 & 1.19 & 407. & 2.14 \\
\hline & 3 & 388. & 1.97 & 402 & 3.82 \\
\hline & 4 & 377. & 4.30 & $\mathrm{~b}$ & 4.37 \\
\hline \multirow[t]{4}{*}{6} & 1 & 1100 & 0.37 & 425. & 2.17 \\
\hline & 2 & 473. & 0.73 & 350. & 7.01 \\
\hline & 3 & 357. & 1.05 & b & 7.53 \\
\hline & 4 & 347. & 1.54 & - & - \\
\hline \multirow[t]{4}{*}{7} & 1 & 2060 . & 0.51 & 617. & 0.56 \\
\hline & 2 & 1100 & 2.09 & 470 & 3.56 \\
\hline & 3 & 488. & 9.59 & b & 4.09 \\
\hline & 4 & 466. & 26.44 & - & - \\
\hline \multirow[t]{4}{*}{8} & 1 & 2360 & 0.61 & 511. & 0.62 \\
\hline & 2 & 1350. & 2.17 & 359. & 2.82 \\
\hline & 3 & 491. & 11.63 & b & 3.52 \\
\hline & 4 & c & 33.80 & - & - \\
\hline \multirow[t]{4}{*}{9} & 1 & 2420 . & 0.48 & 626. & 0.72 \\
\hline & 2 & 878 & 1.49 & 476 . & 5.07 \\
\hline & 3 & 475 & 6.94 & b & 5.62 \\
\hline & 4 & 471 & 13.52 & - & - \\
\hline
\end{tabular}

${ }^{\mathrm{a}} \mathrm{CPU}$ seconds

${ }^{\mathrm{b}}$ No improvement

'Iteration limit exceeded

not degrade as problem size increases. The EM bound times, however, increase greatly as additional random variables are included into the problem. The true advantage of the sublinear method is, therefore, in problems with greater numbers of random variables, as is evident in Problems 7-9. Here, the sublinear method obtains relatively good bounds (within $25 \%$ of the best lower bound $\mathrm{J}$ ) in fractions of the times for NDSP. 
The sublinear method implemented here also solves a smaller number of linear programs than the EM bound. In these examples, the fourth iteration EM bound requires five linear programs for Problems 1 to 3, twenty-five linear programs for Problems 4 to 6 , and one hundred twenty-five linear programs for Problems 7 to 9. In SUB, these sets of problems involved three, nine and twenty-seven linear programs, respectively, of the same size as in EM. These comparisons are meant just to give another example of the work in the implementations explored here. The linear programs, however, involve different constraints in the two procedures and cannot be equated.

\section{Discussion and extensions}

The results in Section 6 indicate that the sublinear upper bounding method efficiently provides good upper bounds on some stochastic programming examples. The sublinear upper bound can also be applied when many random variables are present. This ability distinguishes the sublinear bound from other approaches. The sublinear bound, however, only majorizes the optimal objective value. Lower bounds, obtained from the Jensen inequality or other outer linearization approach (see Marti, 1975; Birge and Wets, 1986a) should be used in a full optimization procedure.

A further refinement of the bound for the recourse function value can be obtained by evaluating the recourse function with respect to groups of random variables. As the number of random variable in these groups increases, the bound is tightened. This procedure is, of course, only useful as long as the integrals are simple enough to allow easy calculation. For example, suppose $m_{2}=3$,

$$
\begin{aligned}
\psi_{2}(\chi, \xi) & =\min \left\{q y \mid W y=\left(\xi_{1}-\chi_{1}, \xi_{2}-\chi_{2}, 0\right)^{\mathrm{T}}, y \geqslant 0\right\} \\
& =\pi_{1}^{i} \zeta_{1}+\pi_{2}^{i} \zeta_{2}
\end{aligned}
$$

where $\left(\zeta_{1}, \zeta_{2}\right)=\left(\xi_{1}-\chi_{1}, \xi_{2}-\chi_{2}\right) \in R^{i}, i=1, \ldots, r$, and we can evaluate

$$
\Psi_{2}\left(\chi_{1}, \chi_{2}\right)=\sum_{i=1}^{r} \int_{R^{i}}\left(\pi_{1}^{i} \zeta_{1}+\pi_{2}^{i} \zeta_{2}\right) \mathrm{d} P\left(\zeta_{1}, \zeta_{2}\right)
$$

We then combine the approximation in (7.2) with a simple recourse function approximation, $\Psi_{3}\left(\chi_{3}\right)$, evaluated with respect to $\zeta_{3}=\xi_{3}-\chi_{3}$. The result is another upper bound on $\Psi$, where

$$
\Psi(\chi) \leqslant \Psi_{2}\left(\chi_{1}, \chi_{2}\right)+\Psi_{3}\left(\chi_{3}\right) .
$$

Again, convex combinations of approximations of the form (7.3) for every pair of random variables can be used to approximate $\Psi$ from above. The practicality of this approach of course relies upon the possibility to evaluate integrals as in (7.2) efficiently. For large groups of random variables, this effort may negate any advantages from this approximation scheme. Prékopa's (1986) sharp approximations of multiple integrals using integrals with respect to small numbers of random variables is, however, encouraging for this approach. 


\section{Appendix}

The following heuristic argument and small example give some justification for the observation in (3.12). Suppose $\left(x^{0}, \chi^{0}\right)$ is a feasible solution of (1.1) that is near the optimal solution. Suppose $\mathscr{D}$ contains all bases (in $W$ ) that correspond to basic optimal solutions, for any possible pair $(\xi, \chi)$, of the linear program that defines $\psi(\chi, \xi)$, cf. Wets $(1974 b)$ for the Basis Decomposition Theorem. Also, suppose that for some $D_{0} \in \mathscr{D}$,

$$
\operatorname{co}\left\{\Psi_{D}, D \in \mathscr{D}\right\}\left(\chi^{0}\right)=\Psi_{D_{0}}\left(\chi^{0}\right) .
$$

(A convex combination of $\Psi_{D}$ values would, in general, appear above. We consider a single function to simplify the argument.) Given (A.1)

$$
\begin{aligned}
\partial\left\{\cos \Psi_{D}\right\}\left(\chi^{0}\right) & =\partial \Psi_{D_{0}}\left(\chi^{0}\right) \\
& =\int \partial \psi_{D_{0}}\left(\chi^{0}, \xi\right) \mathrm{d} P(\xi) .
\end{aligned}
$$

Observe that $\partial \psi_{D_{0}}\left(\chi^{0}, \xi\right)=-q_{D_{0}}^{+}$if $\xi \in \Xi_{0}$, where

$$
\Xi_{0}=\left\{\xi \mid D_{0}^{-1} \xi \geqslant D_{0}^{-1} \chi^{0}\right\} \text {. }
$$

Since $\Psi_{D_{0}}$ gives the convex hull operation value, the region of $\Xi$ in which $\Psi_{D_{0}}$ is exact should be large. One can then reasonably expect $\Xi_{0}$, or more generally the region surrounding $\Xi_{0}$, to contain most of the probabiliy mass of $\Xi$. Hence the subgradients of $\partial \Psi_{D_{0}}$ at $\chi^{0}$ are approximated by $-q_{D_{0}}^{+}$. On the other hand (Wets, 1974 b, Section 7), excluding possibly some boundary cases,

$$
\begin{aligned}
\partial \Psi\left(\chi^{0}\right) & =\int \partial \psi\left(\chi^{0}, \xi\right) \mathrm{d} P(\xi) \\
& =\sum_{D \in \mathscr{D}}\left(-\pi_{D}\right) P\left[D^{-1} \xi \geqslant D^{-1} \chi^{0}\right]
\end{aligned}
$$

where $\pi_{D}$ are the multipliers associated with the basis $D$. Again, since $\Xi_{0}$ and its neighbors occupy much of the probability mass of $\Xi, \partial \Psi\left(\chi^{0}\right)$ is reasonably well approximated by $-\pi_{D_{0}}$. From construction, $q_{D_{0}}^{+}=\pi_{D_{0}}$. Hence, both $\partial \operatorname{co}\left\{\Psi_{D}\right\}$ and $\partial \Psi$ at $\chi^{0}$ are near $-\pi_{D_{0}}$.

As an example, consider the stochastic program:

find $x, y_{1}^{+}, y_{1}^{-}, y_{2}^{+}, y_{2}^{-}, y_{3} \geqslant 0$ such that

$$
\begin{aligned}
& \quad \begin{array}{rlrl}
x & & & \leqslant 1, \\
t_{1} x+y_{1}^{+}-y_{1}^{-} & & & =\xi_{1}, \\
t_{2} x & +y_{2}^{+}-y_{2}^{-}+y_{3} & =\xi_{2},
\end{array} \\
& \text { to minimize } z=c x+E_{\xi}\left[y_{1}^{+}+y_{1}^{-}+y_{2}^{+}+y_{2}^{-}+y_{3}\right],
\end{aligned}
$$

where $\xi_{i}$ is uniformly distributed on $[0,1]$, for $i=1,2$. The optimal subproblem basis (or the related basis with positive coordinates) is indicated for each region of 
$[0,1] \times[0,1]$ in Fig. 1 . The three bases are

$$
D^{1}=\left(\begin{array}{ll}
1 & 0 \\
0 & 1
\end{array}\right), \quad D^{2}=\left(\begin{array}{ll}
1 & 1 \\
0 & 1
\end{array}\right), \quad D^{3}=\left(\begin{array}{ll}
0 & 1 \\
1 & 1
\end{array}\right),
$$

which we use to develop approximating functions $\Psi_{D^{1}}, \Psi_{D^{2}}$ and $\Psi_{D^{3}}$.

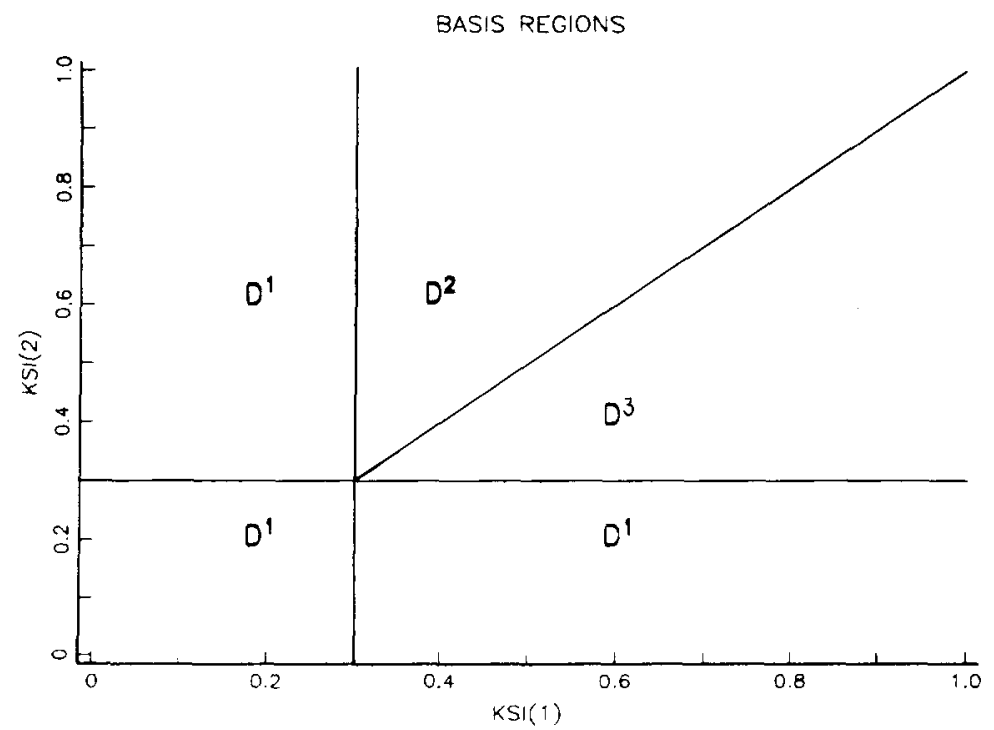

Fig. 1. Optimal subproblem basis regions.

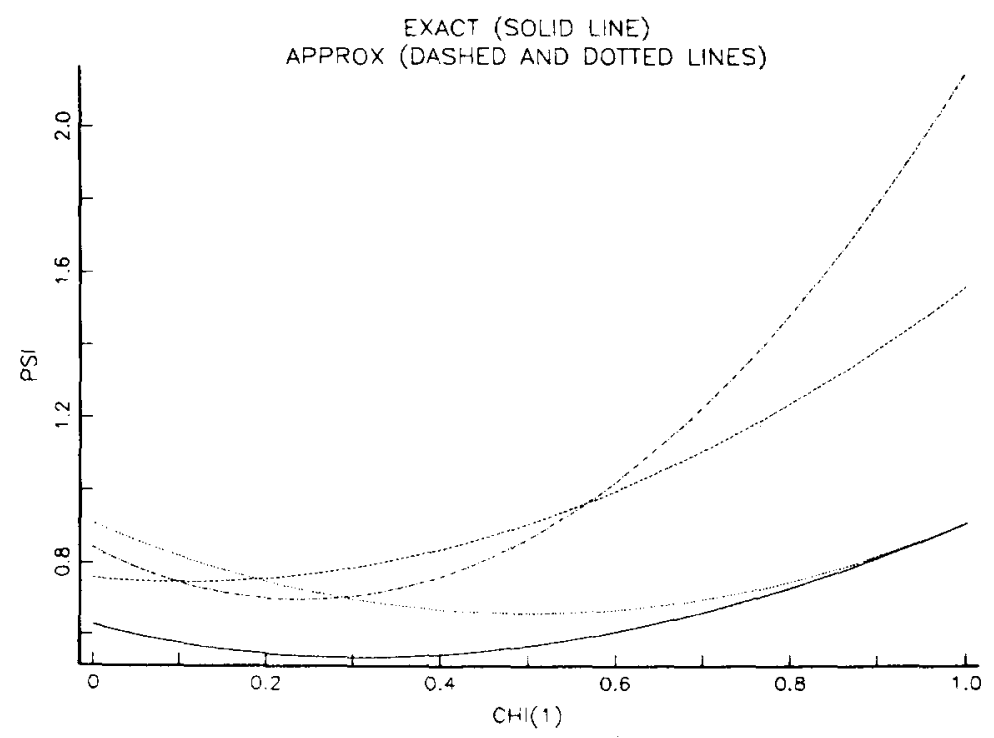

Fig. 2. Comparison of $\psi$ values. 
A comparison of the three approximations and the exact value of $\Psi$ is given in Fig. 2, where $\chi_{2}=0.1$. Note that the slope of the convex hull of the approximating functions closely approximates the slope of $\Psi$. At low values of $\chi_{1}$, the $D^{3}$ and $D^{2}$ optimal region occupy most of $\Xi$ and hence, convex combinations of their subgradients provide good approximations of $\partial \Psi$. At higher values of $\chi_{1}$, the optimal regions for $D^{3}$ and $D^{2}$ diminish, so that $\partial \Psi_{D^{1}}$ provides a good approximation of $\partial \Psi$.

\section{References}

J.R. Birge, "Using sequential approximations in the L-shaped and generalized programming algorithms for stochastic linear programs," Technical Report No. 83-12, Department of Industrial and Operations Engineering, The University of Michigan (Ann Arbor, MI, 1983).

J.R. Birge, "Decomposition and partitioning methods for multi-stage stochastic linear programs," Operations Research 33 (1985) 989-1007.

J.R. Birge, "An L-shaped computer code for multi-stage stochastic linear programs," in: Y. Ermoliev and R. Wets, eds., Numerical Methods in Stochastic Optimization (Springer-Verlag, Berlin, 1987) to appear.

J.R. Birge and R.J-B. Wets, "Approximations and error bounds in stochastic programming," in: Y. Tong, ed., Inequalities in Statistics and Probability, IMS Lecture Notes-Monograph Series Vol. 5 (Institute of Mathematical Statistics, Hayward, CA, 1984) pp. 178-186.

J.R. Birge and R.J-B. Wets, "Designing approximation schemes for stochastic optimization problems, in particular, for stochastic programs with recourse," Mathematical Programming Study 27 (1986) 54-102.

J.R. Birge and R.J-B. Wets, "On-line solution of linear programs using sublinear functions," Technical Report No. 86-25, Department of Industrial and Operations Engineering, The University of Michigan (Ann Arbor, MI, 1986).

J.R. Birge and R.J-B. Wets, "Computing bounds for stochastic programming problems by means of a generalized moment problem," Mathematics of Operations Research 12 (1987) 149-162.

G.B. Dantzig and A. Madansky, "On the solution of two-stage linear programs under uncertainty," in: J. Neyman, ed., Proceedings of the Fourth Berkeley Symposium on Mathematical Statistics and Probability (University of California Press, Berkeley, 1961) pp. 165-176.

Y. Ermoliev, "Stochastic quasigradient methods and their applications to systems optimization," Stochastics 9 (1983) 1-36.

Y. Ermoliev and A. Gaivoronski, "Stochastic quasigradient methods and their implementation," in: Y. Ermoliev and R. Wets, eds., Numerical Methods in Stochastic Optimization (Springer-Verlag, Berlin, 1987) to appear.

A. Geoffrion, "Duality in nonlinear programming: a simplified applications-oriented development," SIAM Review 13 (1971) 1-37.

P.E. Gill, W. Murray and M.H. Wright, Practical Optimization (Academic Press, London and New York, 1981).

C.C. Huang, W. Ziemba and A. Ben-Tal, "Bounds on the expectation of a convex function of a random variable: with applications to stochastic programming," Operations Research 25 (1979) 315-325.

P. Kall, "Computational methods for solving two-stage stochastic linear programming problems," Zeitschrift für Angewandte Mathematik und Physik 30 (1979) 261-271.

P. Kall, K. Frauendorfer and A. Ruszczynski, "Approximation techniques in stochastic programming," in: Y. Ermoliev and R. Wets, eds., Numerical Methods in Stochastic Optimization (Springer-Verlag, Berlin, 1987) to appear.

P. Kall and E. Keller, "Computational experience in solving stochastic programs," Technical Report, Institut für Operations Research, Universität Zürich (Zürich, 1983).

P. Kall and D. Stoyan, "Solving stochastic programming problems with recourse including error bounds," Mathematische Operationsforschung und Statistik Series Optimization 13 (1982) 431-447. 
C. Lemaréchal, "Bundle methods in nondifferentiable optimization," in: C. Lemaréchal and R. Mifflin, eds., Nonsmooth Optimization (Pergamon Press, Oxford, 1978) pp. 79-102.

C. Lemaréchal, J.J. Strodiot and A. Bihain, "On a bundle algorithm for nonsmooth optimization," in: O. Mangasarian, S. Robinson and R. Meyer, eds., Nonlinear Programming 4 (Academic Press, New York, 1981) pp. 245-282.

F.V. Louveaux, "Optimal investment for electricity generation: A stochastic model and a test problem," in: Y. Ermoliev and R. Wets, eds., Numerical Methods in Stochastic Optimization (Springer-Verlag, Berlin, 1987) to appear.

A. Madansky, "Bounds on the expectation of a convex function of a multivariate random variable," Annals of Mathematical Statistics 30 (1959) 743-746.

K. Marti, "Approximationen von Entscheidungsproblemen mit linearer Ergebnisfunktion und positiv homogener, subadditiver Verlusfunktion," Zeitschrift für Wahrscheinlichkeitstheorie und Verwandte Gebiete 31 (1975) 203-233.

B.A. Murtagh and M.A. Saunders, "MINOS/AUGMENTED user's manual," Technical Report SOL 80-14, Systems Optimization Laboratory, Department of Operations Research, Stanford University (Stanford, CA, 1980).

J.L. Nazareth and R.J-B. Wets, "Algorithms for stochastic programs: The case of nonstochastic tenders," Mathematical Programming Study 28 (1986) 1-28.

S.C. Parikh, "Lecture notes on stochastic programming," Unpublished lecture notes, University of California (Berkeley, CA, 1968).

E. Polak, "On the mathematical foundations of nondifferentiable optimization in engineering design," SIAM Review 29 (1987) 21-90.

A. Prékopa, "Boole-Bonferroni inequalities and linear programming," Rutgers Research Report \#4-86, Rutgers Center for Operations Research, Rutgers University (New Brunswick, NJ, 1986).

L. Qi, "An alternating method for stochastic linear programming with simple recourse," Mathematical Programming Study 27 (1986) 183-190.

R.T. Rockafellar, Convex Analysis (Princeton University Press, Princeton, New Jersey, 1970).

R.T. Rockafellar, Conjugate Duality and Optimization (SIAM Publications, Philadelphia, 1974).

R.T. Rockafellar, Network Flows and Monotropic Optimization (Wiley, New York, 1984).

B. Strazicky, "Computational experience with an algorithm for discrete recourse problems," in: M.A.H. Dempster, ed., Stochastic Programming, Proceedings of the 1974 Oxford International Conference (Academic Press, London, 1980) pp. 263-274.

R. Van Slyke and R.J-B. Wets, "L-shaped linear programs with application to optimal control and stochastic programming," SIAM Journal on Applied Mathematics 17 (1969) 638-663.

S.W. Wallace, "A piecewise linear upper bound on the network recourse problem," Mathematical Programming 38 (1987) 133-146.

R.J-B. Wets, "Programming under uncertainty: The complete problem," Zeitschrift für Wahrscheinlichkeitstheorie und Verwandte Gebiete 4 (1966) 316-339.

R.J-B. Wets, "Stochastic programming," Unpublished lecture notes, University of Kentucky (Lexington, $\mathrm{KY}, 1974$ ).

R.J-B. Wets, "Stochastic programs with fixed recourse: The equivalent deterministic problem," SIAM Review 16 (1974) 309-339.

R.J-B. Wets, "Solving stochastic programs with simple recourse, II," in: Proceedings of 1975 Conference on Information Sciences and Systems (Johns Hopkins University, Baltimore, Maryland, 1975).

R.J-B. Wets, "Large scale linear programming techniques in stochastic programming," in: Y. Ermoliev and R. Wets, eds., Numerical Methods in Stochastic Optimization (Springer-Verlag, Berlin, 1987) to appear.

P. Wolfe, "A method of conjugate subgradients for minimizing convex functions," Mathematical Programming Study 3 (1975) 145-173. 